\title{
TCP Performance Measurement in Different GPRS Network Scenarios
}

\author{
A.K. Othman, M. Zakaria, K. Ab. Hamid \\ Universiti Malaysia Sarawak, 94300 Kota Samarahan, Sarawak, Malaysia. \\ Tel: +60 82583323 Fax: +60 82583409 \\ Email: okhalid@feng.unimas.my,muzalina_zakaria@yahoo.com,khair@cans.unimas.my
}

\begin{abstract}
This paper presents the results of measured TCP performance in one of the commercially deployed live GPRS network in Malaysia under various network scenarios based on users' physical locations. End-toend evaluation of FTP application is used for the assessment. Tracing at the GPRS air interface is done simultaneously, using a proprietary GSM/GPRS air interface testing tool and software. The results show that TCP performance is stable in all scenarios and can adapt fairly well to GPRS mobility and bad channel condition, although at the expense of reduced throughput. TCP imposes its slow start/congestion avoidance mechanism in such situations where packet loss is common. Tuning the TCP parameters to optimise its performance also proves to be beneficial.
\end{abstract}

Keywords: GPRS; TCP; Performance; Network Scenarios

\section{Introduction}

General Packet Radio Service (GPRS) [1], [2] is a packet-switched extension of Global System for Mobile communications (GSM) network that offers access to data services such as Internet applications to mobile users anywhere, anytime, as long as they are within its coverage areas. GPRS provides the link between the wireless GSM network and the wired external packet-switched data networks (PDNs) that hosted the Internet applications. It interconnects with these PDNs through Internet Protocol (IP) and this makes GPRS essentially an IP based network entity. With GPRS, the available data rate is between $36.2 \mathrm{kbps}$ to $85.6 \mathrm{kbps}$ with four time slots allocation

Internet applications such as web surfing, e-mail and file transfer rely on Transmission Control Protocol (TCP) [3] as a reliable transport for data transfers. It is a connection-oriented, packet-switched transport method that delivers data in small segments or packets. TCP ensures ordered, error-free data delivery with its sequence numbering and acknowledgment systems together with retransmission of loss packets and checksum evaluation. Additionally, it provides data flow control through its congestion control mechanisms.
TCP is originally designed for data transfers across wired, fixed networks. It anticipates transmission problems that are typical with wired networks behaviours. With the implementation of GPRS, TCP traverses the wireless mobile network which is a different environment from the wired network. Moreover, due to the mobility factor, GPRS users are subjected to several network scenarios based on their physical locations. For example in urban area, common locations for GPRS users are inside buildings, or outdoors in moving vehicles. The performance of Internet applications over live GPRS network as perceived by users may vary depending on these scenarios. This issue has not been specifically addressed in the previous studies conducted on TCP performance in GPRS network [4], [5], [6]. Therefore, to have more conclusive results, TCP performance measurement in different network scenarios ought to be taken under consideration.

This paper evaluates the TCP performance in one of the commercially deployed GPRS networks under different scenarios based on GPRS users' typical locations in urban environment. This is accomplished by incorporating TCP packet captures in GPRS drivetest measurement. TCP tuning is done as well to optimise the performance. The results obtained are only relevant to the particular GPRS network being assessed, thus may not apply to all GPRS networks in general.

The TCP performance metrics such as throughput and packet loss are examined together with GPRS tracing at the air interface. The results show that on average, the TCP performance is stable in all scenarios. TCP adapts fairly well to the GPRS mobility and bad channel condition despite low throughput.

The rest of the paper is organised as follows: section 2 describes network scenarios selected for the measurement. Section 3 explains on GPRS parameters setup as implemented in the commercial network. Section 4 gives a brief outline on TCP tuning. In section 5, measurement set up is presented. The results obtained are discussed in Section 6 . 